\title{
Total Cost of Ownership bei Prozessleitsystemen
}

\author{
Wie sich die Kostenaspekte entwickelt haben
}

Wer sich im Rahmen von NAMUR-Aktivitäten mit diesem Thema beschäftigt, muss sich natürlich mit der NAMUR-Empfehlung „Qualitätssicherung leittechnischer Systeme“, der NE 121, auseinander setzen.Das Ziel dieser NAMUR-Empfehlung ist die Qualitätssicherung und das Veralterungsmanagement leittechnischer Systeme über den gesamten Lebenszyklus, wobei mit leittechnischen Systemen das gesamte Spektrum von der Feldebene über das Prozessleitsystem bis hin zur MES-Ebene (Manufacturing Execution System) gemeint ist. Dieser Beitrag baut auf vielen grundsätzlichen Aussagen der NE 121 auf, betrachtet aber lediglich Prozessleitsysteme und beschäftigt sich in diesem Zusammenhang vor allem mit Kostenaspekten. Der NAMUR AK 2.1 (SPS und PLS) hat sich in den letzten Sitzungen auch gemeinsam mit PLS-Lieferanten diesem Thema angenommen. Über die Ergebnisse dieser Diskussionen wird hier berichtet.

SCHLAGWÖRTER Prozessleitsystem / Kostenentwicklung / TCO

\section{Total Cost of Ownership for Distributed Control Systems}

When evaluating the subject "TCO for Distributed Conrol Systems" the NAMUR recommendation (NE) 121 "Quality Assurance of Control Systems" has to be taken into account. The objective of this NAMUR recommendation is the utilisation of quality assurance and obsolescence management of instruments for the entire life cycle of industrial process control systems, where the term process control system covers the field level, the distributed control system (DCS) as well as the MES level (manufacturing execution system). This presentation is based on several fundamental statements of the NE 121, however it just concentrates on distributed control systems and in this context it essentially pays attention to different cost aspects. The NAMUR working group AK 2.1 (PLC and DCS) was - in its recent meetings working on this topic. The results of the discussions, where DCS manufacturers were also involved, are presented in this contribution. 
WOLFGANG ALBERT, NAMUR Arbeitskreis 2.1 (SPS und PLS) / Evonik Degussa

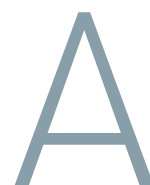

ls der NAMUR Arbeitskreis 2.1 (SPS und PLS) vor etwas mehr als zwei Jahren in einem „Brainstorming" eine Liste mit neuen Arbeitsthemen aufstellte, war unter anderem auch der Komplex „PLS-Kosten“ dabei. Schließlich wurde aus diesem Arbeitstitel das offizielle Thema „TCO (Total Cost of Ownership) bei Prozessleitsystemen“ [2]. Wir waren uns innerhalb des Arbeitskreises relativ schnell darüber im Klaren, dass sich die PLS-Technologie in den letzten 15 - 20 Jahren grundlegend geändert hat. Bei den Aussagen zu PLS-Anschaffungs- oder -investitionskosten und PLS-Instandhaltungskosten wurden zwar spontane Mehrheiten in Richtung „,Kostenänderung sprich Kostenreduzierung“ erzielt, aber keine Einstimmigkeit. Es gab auch Statements mit dem Inhalt ,eigentlich hat sich so viel gar nicht geändert". Dies war dann der Anstoß, sich mit PLS-Lieferanten zusammen zu setzen und die Themen mit den Lieferanten, aber auch arbeitskreis-intern im Detail zu diskutieren. Wie Bild 1 zeigt, beteiligten sich auf Anwenderseite die im Arbeitskreis vertretenen NAMUR-Mitgliedsfirmen sowie auf Lieferantenseite eine Großzahl der im Bereich Prozessautomatisierung etablierten PLS-Hersteller.

Nach einer Beschreibung der Herausforderungen, die sich bei der Bearbeitung eines derartigen Themas stellen, und der Definition der Randbedingungen setzt sich der Kern des Beitrags mit zwei Fragestellungen auseinander: „Wie haben sich die PLS-Beschaffungskosten in den letzten 15 - 20 Jahren entwickelt?“ und „Welche Größenordnung erreichen die Life Cycle Kosten, wenn man jetzt ein PLS beschafft und dieses über einen Zeitraum von wiederum 15 - 20 Jahren betreibt?“. In der Zusammenfassung wird auch die besondere Rolle der NAMUR bei den zuvor beschriebenen Entwicklungen beschrieben.

Wer sich im Rahmen von NAMUR-Aktivitäten mit dem Thema „PLS-Kosten“ beschäftigt, muss sich natürlich auch mit der NAMUR-Empfehlung „Qualitätssicherung leittechnischer Systeme“, der NE 121 [1] auseinander setzen. Das Ziel dieser NAMUR-Empfehlung ist die Qualitätssicherung und das Veralterungsmanagement leittechnischer Systeme über den gesamten Lebenszyklus, wobei mit leittechnischen Systemen hier das gesamte Spektrum von der
Feldebene über das Prozessleitsystem bis hin zur MESEbene (Manufacturing Execution System) gemeint ist.

Dieser Beitrag baut auf vielen grundsätzlichen Aussagen der NE 121 auf und beschäftigt sich mit folgenden Fragen: Wie sind die Innovationsraten und wie sind die Stabilitätsanforderungen in den verschiedenen Ebenen verteilt? Wie sieht es mit den Lebenszyklen in den Ebenen aus? Er betrachtet aber lediglich Prozessleitsysteme (Bild 2), also nicht die Feldebene und nicht den Bereich MES (Manufacturer Execution System), und beschäftigt sich vor allem mit Kostenaspekten.

\section{HERAUSFORDERUNGEN}

Wie schon erwähnt, sollen die Hauptthemen dieses Beitrags Kosten und Kostenentwicklungen sein. Die besondere Herausforderung bei der Bearbeitung derartiger Themen ist, dies firmenübergreifend zu tun und gleichzeitig die Vertraulichkeit der firmenspezifischen Gegebenheiten zu wahren.

Bei Prozessleitsystemen werden Investitionskosten zur Erstbeschaffung dieser Systeme nach einer vernünftigen Ausschreibung in der Regel akzeptiert. Anders sieht es aber bei Kosten aus, die aufgrund von Migrationen und von Revisionswechseln anfallen. Die Notwendigkeit dieser Ausgaben wird immer wieder heftig diskutiert und in Frage gestellt. Auch wohl deshalb, weil für den Anlagenbetreiber bei diesen Ausgaben ein Nutzen in klassischem Sinn nicht zu erkennen ist. Man erhält durch diese Aktionen in der Regel kein besseres Produkt. Und der Erhalt der Funktionsfähigkeit des Prozessleitsystems zählt in vielen Fällen nicht zur klassischen Nutzenkategorie.

Um die Betriebsphase eines Prozessleitsystems zu beschreiben, muss zunächst einmal definiert werden, was zur PLS-Instandhaltung gehört. Die Beantwortung der beiden Fragen, ob die notwendigen Instandhaltungsaufwendungen vom PLS-Hersteller auf der einen Seite oder von Art und Größe des Prozesses auf der anderen Seite abhängen, ist nicht einfach. Einstimmige Antworten sind hier in einer Gruppe - wenn überhaupt - erst nach längeren Diskussionen zu finden. 


\section{RANDBEDINGUNGEN}

Zunächst wurde festgelegt, dass der Investitionkostenvergleich zwischen früher und heute auf Systemen mit zumindest ähnlicher Architektur basieren sollte. Dies sollte einen Vergleich möglichst wenig beeinträchtigen und bedeutete, dass neuere Technologien, die sich kostenmäßig in der einen oder anderen Richtung auswirken können, nicht berücksichtigt wurden. Verbesserungen bei der Erstellung der Applikationssoftware (z.B. ein eingeführtes oder verbessertes „Bulk-Engineering“), die im Laufe der Zeit eingeführt wurden, sind dagegen berücksichtigt. Ebenfalls berücksichtigt wurde die Tatsache, dass Prozessnahe Komponenten (PNK, Controller) immer leistungsfähiger wurden und deshalb die notwendige Anzahl dieser Einheiten in gleichem Maße reduziert werden konnte.

Die Kosten wurden in 3 Kategorien aufgeteilt.

- Hardware und Software

- Applikationssoftware (Konfiguration)

- Zukaufteile

Bei der ersten Kategorie ist unter Software nicht die Applikationssoftware, d.h. die Konfiguration gemeint. Hier werden die Lizenzkosten für die Systemsoftware erfasst. Die Aufteilung dieser Kategorie in zwei separate war nicht möglich, da bei älteren Systemen die getrennte Angabe der beiden Kostenblöcke nicht üblich war.

Die zweite Kategorie umfasst alle Aufwendungen, um die anlagenspezifische Automatisierungsstrategie (Abläufe, Verriegelungen, Regelungen etc.) im System abzubilden, und der dritte Block betrifft die notwendigen Zukaufteile.

Die Kosten wurden - so gut es ging - über die letzten 15 - 20 Jahre ermittelt und werden einmal ohne Inflation und zum anderen mit einer Inflationsrate von $2 \%$ pro Jahr angegeben. Da niemand in der Prozessindustrie Listenpreise für Prozessleitsysteme bezahlt, wurde ein Standardrabatt von $20 \%$ angenommen - so realistisch oder unrealistisch dieser Rabattsatz aus individueller Firmensicht auch sein mag. Auch wurden eine Standardsystemgröße und ein System-Layout definiert (Bild 3). Um die Komplexität einzuschränken, wurde hierbei ein kontinuierlicher Prozess betrachtet.

Die Anzahl der I/O's, nämlich 2.250, ist nicht das Ergebnis langer Überlegungen und Diskussionen, sondern es war ganz einfach so, dass der erste PLS-Lieferant, der im Vorfeld unserer Betrachtungen eine Kostenabschätzung abgab, diesen Umfang wählte und dass dieser Umfang dann - der Einfachheit halber - für alle anderen Lieferanten übernommen wurde. Um - bezogen auf das vorgegebene System-Layout - auf PLS-Kosten aus der Vergangenheit zu kommen, wurden bei den PLS-Herstellern die noch verfügbaren Angebotssoftware-Module aus den jeweiligen Zeiträumen benutzt.
Bei den Betreuungskosten, den Maintenance-Kosten, wurden

- Konfigurationsanpassungen bei z.B. Anlagenänderungen

- Trainingsaufwendungen

- Implementierung von „Advanced Tools“

nicht berücksichtigt. Die Notwendigkeit dieser Ausgaben wird auch ganz selten diskutiert, weil sie unmittelbar eingesehen wird oder weil den Ausgaben auch ein direkter Benefit gegenüber steht und sich demzufolge eine nachvollziehbare Rendite berechnen lässt.

Folgende typische Maintenance-Kosten wie

- Servicevertrag (Ersatzteilhaltung, Hot-Line-Service, Rufbereitschaft)

- Software- und Hardware-Upgrade

- (Systemupgrade und Austausch aller Standard-Hardware-Komponenten alle 5-6 Jahre)

sind natürlich berücksichtigt. Warum sich mit den letzten beiden Kostengruppen so gut wie niemand direkt abfinden will, wird in den nachfolgenden Kapiteln noch näher erläutert. Die Angabe der Betreuungskosten erfolgt immer in Prozent und bezieht sich auf die Anschaffungskosten des Prozessleitsystems.

\section{PLS-KOSTENENTWICKLUNG}

Den ersten Kernpunkt des Beitrags stellt das Thema „PLSKostenentwicklung“ dar. Die Angabe über Veränderungen innerhalb der letzten Jahre ist natürlich mit einer gewissen Unschärfe versehen. Deshalb werden nur Bereiche angegeben, innerhalb derer sowohl die Angaben der PLSLieferanten als auch die Angaben der Anwender im Wesentlichen untergebracht werden können.

Die Anschaffungskosten für Prozessleitsysteme gingen (ohne Inflation) innerhalb der letzten 15 - 20 Jahre um 30 - 45\% zurück, wobei dies zu einem Gutteil auf die erste Kostenkategorie, die Hard- und Softwarekosten, zurückzuführen ist. Die Kategorien „Zukaufteile“ und „Engineering“, d.h. die Erstellung der Konfiguration (Applikationssoftware) gehen hier nicht so stark ein.

Unter Berücksichtigung einer Inflationsrate von $2 \%$ pro Jahr ergibt sich eine Kostenreduzierung von $45-$ $60 \%$. Bei Berücksichtigung der Inflationsrate gehen nun auch die Kosten für die Zukaufteile zurück.

In Bild 4 ist aber nur „ein Teil der Wahrheit“ zu sehen. Was bisher bei der Ermittlung der Kostentrends keine Berücksichtigung fand und was auch unter Anwenderfirmen nur sehr „nebulös“ diskutiert werden kann, ist die Entwicklung von Nachlässen, von Rabatten auf Listenpreise. In unserer Betrachtung wurde ein konstanter Rabattsatz von $20 \%$ angenommen. In der Realität ist diese Konstanz nicht gegeben. Innerhalb 
Folgende PLS-Lieferanten waren involviert:

SIEMENS
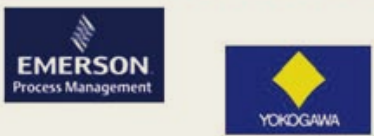

Honeywell



Folgende Anwenderfirmen waren involviert:

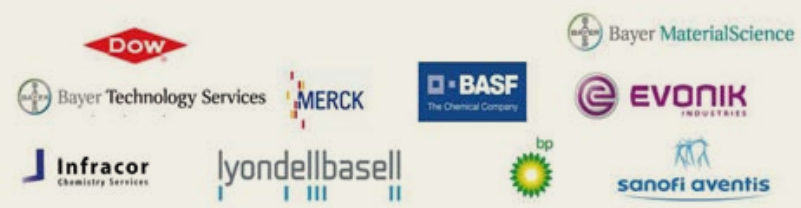

BILD 1: Beteiligte PLS-Lieferanten und Anwenderfirmen

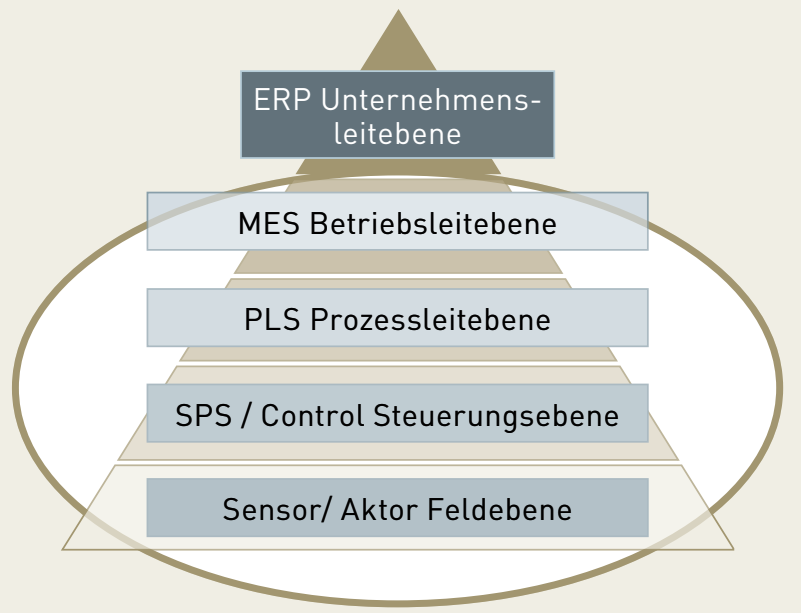

BILD 2: Betrachtete Automatisierungsebenen

Investitionskosten

System Architektur (Konti-Prozess, nicht reguliertes Umfeld):

$2.250 \mathrm{l} /$ Os (800 analog, 1.450 binär, 1.000 Ex-Signale)

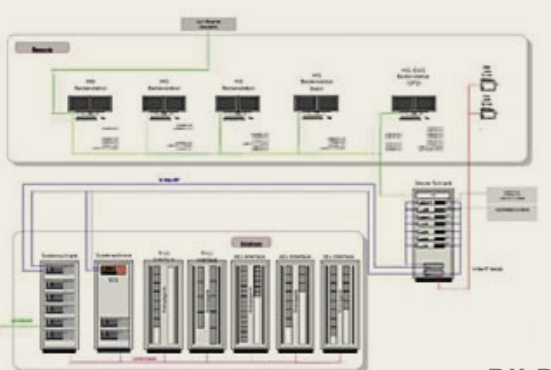

$2-n$ PNK's

4 Bedienplätze

1 Engineering

Station

BILD 3: Systemlayout

\section{Ohne Inflation}



Mit Inflation (2\% pro Jahr)

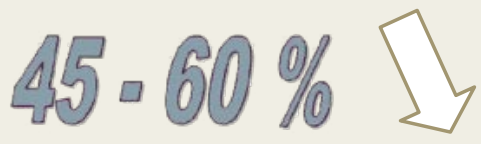

BILD 4: PLS-Kostenentwicklung über die letzten 15 - 20 Jahre 
des NAMUR AK 2.1 konnten wir uns zumindest darauf einigen, dass die Nachlässe im Laufe der Zeit prozentual nach oben gegangen sind, so dass diese Angaben hier ein eher vorsichtiges, konservatives Bild abgeben. Die grafisch dargestellten Trends der Kostenentwicklung - hier ohne Inflationseinflüsse - sind aus Bild 5 zu entnehmen.

Was aus dieser Darstellung hervorgeht, ist, dass sich signifikante Technologiesprünge auch preismäßig aus- wirken. So ist z.B. die Einführung der PC-Technologie um die Jahrtausendwende „sehr schön“ sichtbar. Hier sind die Kostentrends eines PLS-Herstellers dargestellt. Wenn von Listenpreisen und Standardrabatten ausgegangen wird, dann unterscheiden sich die Trends anderer Lieferanten nur wenig von dem hier Gezeigten. Auch spielt die Größe des Prozesses für die grundlegenden Kostenentwicklungen überhaupt keine Rolle. Spezifische Kosten wie z.B. Kosten pro Ein-/Ausgang gehen natürlich



\section{Beispiel für Life Cycle Kosten}

Kleinere Software Upgrades alle 3 Jahre

Größere Software und Hardware Upgrades alle 6 Jahre

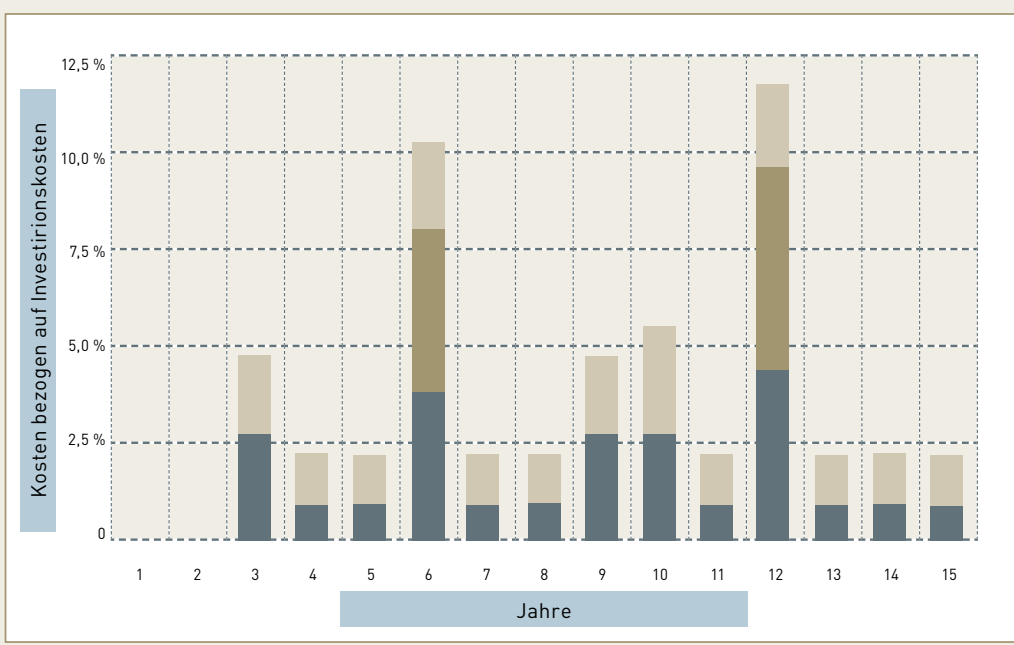

Kostenentwicklung (Beispiel PLS-Hersteller)

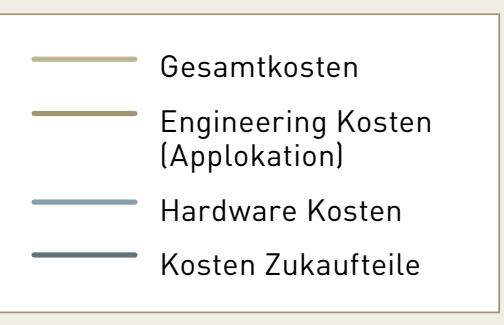

BILD 5:

Grafische Darstellung der Kostentrends (ohne Inflation)

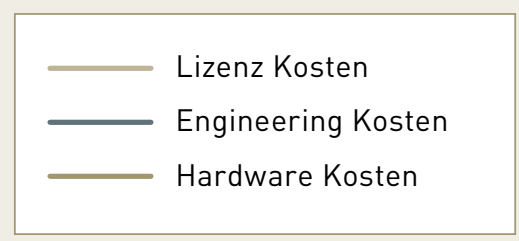

BILD 6:

PLS Life Cycle Kosten über einen Zeitraum von 15 Jahren 
mit zunehmender Systemgröße zurück. Das war früher so und ist heute noch genauso.

\section{PLS LIFE CYCLE KOSTEN}

Was kostet denn nun ein Prozessleitsystem im Betrieb? Was sind die Life Cycle Kosten (wobei hier die Betriebsoder Maintenancekosten gemeint sind)? Zunächst bieten PLS-Lieferanten Wartungsverträge in unterschiedlichsten Ausprägungen an, d.h. mit unterschiedlichen Leistungen und Reaktionszeiten. Die Vetragsgestaltung und demzufolge auch die Vertragskosten hängen natürlich auch von Umfang und Qualität einer „Inhouse“Wartung ab. Als grober Anhaltspunkt kann für die Wartungskosten ca. 2 - 4\% der Anschaffungskosten pro Jahr angenommen werden.

Ein Systemupgrade zusammen mit dem Austausch von Standard-Hardwarekomponenten beläuft sich auf ca. $10 \%$ der Investitionskosten, so dass bei einem UpgradeZyklus von 5 Jahren jährlich nochmals $2 \%$ anfallen.

Bei jährlichen mittleren Kosten von 4 - 6\% (hier sind die beiden obigen Zahlen einfach addiert) müssen 60 90\% der Investitionskosten über einen Zeitraum von 15 Jahren nochmals ausgegeben werden. Oder anders ausgedrückt: Die Anschaffungskosten für ein Prozessleitsystem fallen für die Betreuung im Laufe von 15 - 20 Jahren nochmals an. Diese Aussage deckt sich auch mit der Angabe in der NAMUR-Empfehlung „Qualitätssicherung leittechnischer Systeme“, der NE 121.

Beispielhaft kann ein Kostenverlauf für ein Prozessleitsystem über 15 Jahre wie in Bild 6 aussehen. Hier werden alle 3 Jahre kleinere Softwareupdates vorgenommen. Die Standardhardware wird alle 6 Jahre ausgetauscht, wobei darüber hinaus nach 12 Jahren auch die Netzwerkkomponenten ausgetauscht werden. Deshalb sind die Kosten hier etwas höher. Die ersten beiden Betriebsjahre fallen unter die Kategorie „Garantiezeit“.

Um potenzielle Betriebskosten schon in der Beschaffungsphase vergleichen zu können, müssen natürlich exakte Vorgaben für alle Anbieter gelten: Welche Wartungsverträge sollen angenommen werden und in welchen Zyklen sind welche Arbeiten (im Wesentlichen Hard- und Software-Upgrades) durchzuführen?

Bei den Anwenderfirmen bestehen natürlich umfangreiche Erfahrungen, die bei bereits durchgeführten PLS-Migrationen gesammelt wurden. Starke, kostenmäßige Variationen bei Hardware und Lizenzen sind hier weniger zu beobachten. Anders sieht es bei einer eventuellen Überarbeitung der Anwendersoftware, der Konfiguration, aus. Mal ist der Aufwand vernachlässigbar oder nicht existent, hin und wieder ist er signifikant. Zumindest aus Sicht der PLS-Hersteller sind signifikante Aufwendungen singuläre Ereignisse und werden wohl in Zukunft nicht mehr vorkommen. Und diese aus der Vergangenheit eventuell bekannten Gege- benheiten werden in der Angebotsphase auch mit Sicherheit (von Seiten der Hersteller) nicht in irgendwelche Kostenprognosen für den zu betrachtenden Betriebszeitraum einfließen.

Die Frage bleibt trotzdem, wie diese eventuellen „Altlasten“ in die Abschätzung der TCO (Total Cost of Ownership) auf Anwenderseite einfließen sollten. Die NAMUR kann hier keine direkten Leitlinien angeben, sie kann nur darauf hinweisen, dass über diesen Aspekt zumindest nachgedacht werden sollte.

Eine weitere interessante Fragestellung, die während der Arbeitskreis-Diskussionen aufkam, war: Kann es sein, dass vor dem Hintergrund der Kostenreduzierung bei den PLS-Investitionen diese Einsparungen im Laufe der Betriebszeit über jährliche Mehrausgaben im Vergleich zu früher wieder kompensiert werden?

Nur ein PLS-Lieferant verfügte über Instandhaltungskosten, wie sie vor 15 Jahren anfielen. Nach diesen Angaben nahmen die Betreuungskosten in ähnlicher Form ab wie die Beschaffungskosten. Ein plausibles Argument dafür ist, dass auch früher Hardwarekomponenten ausgefallen sind und ausgetauscht werden mussten, dass aber in früheren Zeiten hierfür wesentlich höhere Kosten als heute anfielen. Vielleicht erinnern sich noch einige der erfahreneren Ingenieure an Zeiten, als für eine PLS-Bedieneinheit DM 150.000 bezahlt werden musste. Bei den beteiligten Anwenderfirmen waren leider keine Zahlen verfügbar, die diese Verhältnisse untermauerten.

Wenn von der Annahme ausgegangen wird, dass sich sowohl die Investitions- als auch die Betriebskosten in den letzten beiden Jahrzehnten mehr als halbiert haben, warum trifft man dann relativ häufig auf Klagen und Ärger über die aktuelle Systemlandschaft - nach dem Motto: Früher war alles besser. Die Klagen werden immer auf den Punkt gebracht, dieser Punkt hat einen Namen und dieser Name ist „Bill Gates“. Dabei hat der von Bill Gates generierte Standard, also PC's und Windows, auch einen Anteil an der Kostenentwicklung. Über die prozentuale Größenordnung dieses Anteils kann sicherlich diskutiert werden. Sollte man, statt zu klagen, nicht lieber die Leistungen dieses Mannes honorieren? Das Unwohlsein, das viele in diesem Zusammenhang beim Namen „Bill Gates“ befällt, hat sicherlich auch psychologische Ursachen.

Früher wurden Teile in der Regel nur dann ausgetauscht, wenn sie defekt waren. Dies hat keine Diskussionen entfacht, weil alle Beteiligten unmittelbar einsahen, dass die Reparatur- oder Ersatzinvestitionen unbedingt notwendig waren, um die Produktionsanlage weiter in Betrieb zu halten.

Heute werden funktionierende Systeme ,prophylaktisch“ ersetzt, und dabei kann nicht einmal angegeben werden, mit welchen Wahrscheinlichkeiten eines Ausfalls, also mit welchen Risiken wir es zu tun haben, wenn wir den Austausch von Systemen über einen län- 
geren Zeitraum hinauszögern. Die einzige Aussage, die seriös zu machen ist, ist, dass die Risiken wohl zunehmen werden. Und dieser Sachverhalt ist von allen Seiten nur schwer zu akzeptieren.

Klar ist, dass eine Systemmigration in erster Linie dem Erhalt der Funktionsfähigkeit dient und kaum einen direkten, produktionsorientierten Nutzen in Form von Kapazitätserhöhungen, Qualitätsverbesserungen oder Energiereduzierungen generiert. Eine Idee, über die nachgedacht werden kann und sollte, bezieht sich darauf, dass Migrationsprojekte mit Produktionsoptimierungsprojekten verknüpft werden können. Mit relativ geringem Mehraufwand lässt sich so in der Regel ein Nutzen in klassischem Sinne aufweisen, der die Genehmigung von Migrationsvorhaben zumindest erleichtern kann.

Die Anforderungen an die Hersteller sind einfach zu formulieren, da es sich auch nur um eine Anforderung handelt:

Jede PLS-Produktentwicklung sollte so gestaltet werden, dass eine einmal erstellte Konfiguration auf allen Nachfolgeversionen ohne manuelle Anpassungen lauffähig ist. Was man dann mit Möglichkeiten macht, die eine neue Version bietet, ist eine ganz andere Geschichte.

Wie bereits geschildert, werden bei Prozessleitsystemen die Investitionskosten im Laufe von 15 - 20 Jahren nochmals für Betreuungsaufwendungen ausgegeben. Um diese Kennzahl zu relativieren, kann man auch in einen anderen Bereich, z.B. die IT (die Informationstechnologie) schauen und für diesen Bereich ausrechnen, wie lange es dort typischerweise dauert, bis die zugeordneten Investitionskosten für Instandhaltungsaufwendungen nochmals ausgegeben sind.

In der IT-Landschaft sind dies maximal 1 - 2 Jahre, was zumindest die vage Aussage rechtfertigt, dass sich die
Maintenance-Kosten in der Prozessautomatisierung in einer vernünftigen Bandbreite befinden.

\section{ZUSAMMENFASSUNG}

In den letzten beiden Jahrzehnten sind die Investitionsund Betreuungskosten bei Prozessleitsystemen signifikant zurückgegangen. Dies ist sicherlich zu einem Gutteil darauf zurückzuführen, dass man sich auf Herstellerseite (auch in Absprache mit den Anwendern) dazu entschlossen hat, den Einsatz von Standardhardware- und -software deutlich zu erhöhen.

Mitbegleitet hat diese Entwicklung sicher auch das „Benutzersprachrohr“, das über die NAMUR oder andere Verbände den veröffentlichten Aussagen, Anforderungen und Empfehlungen die notwendige Einflusskraft verleiht und in diesem Fall mit dafür gesorgt hat, dass der vermehrte Einsatz von Standards so gestaltet wird, dass nicht nur die Beschaffungskosten reduziert wurden, sondern dass die Robustheit der Systeme nicht zu stark unter den Standardisierungsaktivitäten leidet. Eine Mitarbeit in diesen Verbänden lohnt sich also definitiv.

MANUSKRIPTEINGANG 27.11.2009

Aus dem NAMUR AK 2.1 haben an diesem Beitrag mitgearbeitet: Eduardus Beullens - Bayer MaterialScience, Dietmar Güldner DOW, Ingrid Hartmann-Lotsch - Infracor, Harry Klein - LyondellBasell, Peter Lotz - Merck, Björg Otte - BP, Ulrich Schlagowski Bayer Technology Services, Peter Schlemmer - BASF, Heinrich Schmedding - Bayer MaterialScience, Wolfgang Thoma - Actemium, Peter Unger - Sanofi-Aventis

\section{AUTOR}

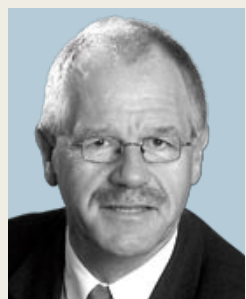

Dipl. Ing. WOLFGANG ALBERT (geb. 1956) leitet bei der Evonik Degussa GmbH das Fachgebiet „Automation und Prozess Management" im Servicebereich Verfahrenstechnik und Engineering.

Evonik Degussa $\mathrm{GmbH}$, Rodenbacher Chaussee 4, 63457 Hanau, Abt. DG-TE-EN-EA2,

Tel. +496181594905 , E- Mail: wolfgang.albertaevonik.com

\section{REFERENZEN}

[1] NAMUR Empfehlung NE 121: Anforderung an Systeme für anlagennahes Asset Management (2001)

[2] Präsentationen zum Thema „TCO (Total Cost of Ownershipl bei Prozessleitsystemen“ von ABB, Emerson, Honeywell, Siemens und Yokogawa 